\title{
ILCEA
}

Revue de l'Institut des langues et cultures

d'Europe, Amérique, Afrique, Asie et Australie

$12 \mid 2010$

La FASP : dix ans après...

\section{La FASP universitaire autour de La Dame Noire}

Entretien avec Tony Gheeraert par Jean-Louis Trouillon

\section{Tony Gheeraert et Jean-Louis Trouillon}

\section{(2) OpenEdition}

1 Journals

Édition électronique

URL : https://journals.openedition.org/ilcea/579

DOI : $10.4000 /$ ilcea. 579

ISSN : 2101-0609

Éditeur

UGA Éditions/Université Grenoble Alpes

Édition imprimée

ISBN : 978-2-84310-180-9

ISSN : 1639-6073

\section{Référence électronique}

Tony Gheeraert et Jean-Louis Trouillon, « La FASP universitaire autour de La Dame Noire », ILCEA [En ligne], 12 | 2010, mis en ligne le 05 octobre 2010, consulté le 02 mai 2022. URL : http://

journals.openedition.org/ilcea/579; DOI : https://doi.org/10.4000/ilcea.579

Ce document a été généré automatiquement le 2 mai 2022.

(C) ILCEA 


\title{
La FASP universitaire autour de $L a$ Dame Noire ${ }^{1}$
}

\author{
Entretien avec Tony Gheeraert par Jean-Louis Trouillon
}

Tony Gheeraert et Jean-Louis Trouillon

Une Dame Noire pour un roman noir. Une écriture en spirale pour un roman à l'intrigue contemporaine mais qui nous renvoie sans cesse aux heures sombres de la Révolution anglaise du XVII ${ }^{\mathrm{e}}$ siècle. Dans son premier roman Tony Gheeraert joue habilement sur plusieurs niveaux de lecture. Est-ce un roman policier? sans aucun doute et il est d'ailleurs publié aux Éditions du POLAR! Mais c'est aussi un roman d'aventure moderne, c'est un roman historique particulièrement bien documenté, c'est un roman qui fourmille d'allusions littéraires que le lecteur cultivé adorera retrouver. C'est aussi, enfin, un roman «clin d'oeil» qui vogue pour mieux s'en moquer sur la mode de ces ouvrages à succès qui se situent à mi-chemin entre l'ésotérique relativement sérieux et le charlatanisme le plus éhonté. En bon critique littéraire qu'il est, Tony Gheeraert s'est amusé à dresser une liste de toutes les ficelles qu'utilisent les auteurs professionnels de ces œuvres, puis il s'est lancé un défi, celui de tresser tous ces brins épars pour composer un ouvrage qui procure un grand plaisir de lecture. Le résultat est convaincant, il faut lire La Dame Noire.

1 JLT : Tony Gheeraert, si nous avons le plaisir de vous accueillir parmi nous aujourd'hui ce n'est pas uniquement parce que vous êtes un collègue, Normalien, Agrégé de Lettres Classiques et Maître de Conférences spécialiste de Littérature française du XviI ${ }^{\mathrm{e}}$ siècle à l'université de Rouen à Mont St Aignan.

Non, ce qui justifie votre présence c'est une autre facette de votre personnalité, vous êtes en effet l'auteur d'un roman en deux volumes, La Dame noire, publié aux Éditions du Polar et qui, dans son traitement, se rapproche très fortement des ouvrages de fiction à substrat professionnel qui font l'objet de nos travaux depuis maintenant dix ans.

Je vous propose d'aborder dans cet entretien votre rapport à l'écriture dans le cadre des préoccupations de notre groupe de recherche, et en particulier les objectifs que vous vous étiez fixés dans votre entreprise et les moyens que vous avez mis en œuvre pour y parvenir. 
Vos lecteurs ordinaires, je veux parler de ceux qui ne sont ni spécialistes de FASP ni de roman académique et qui s'expriment sur des blogs divers, font des allusions au $D a$ Vinci Code. Vous-même citez dans votre ouvrage Le Nom de la rose, vous citez également Mrs Radcliffe, vous pensiez sans doute mais sans le dire à The Italian. À mon avis ces critiques n'ont pas compris votre esprit frondeur que vous tenez certainement de votre spécialité de dix-septiémiste, et le fait que vous ne citiez pas le Northanger Abbey de Jane Austen aurait tendance à me renforcer dans mon idée qu'il faut aller au-delà des apparences que vous présentez de façon évidente. Alors, qui a raison, eux ou moi ? Quel est votre message? Recherchez-vous une mission sociétale? Est-ce que vous voulez être, avec ce policier historique, le Cervantes qui a tué les romans de chevalerie et empêcher ainsi l'écriture d'un autre Da Vinci Code?

2 TG : Je vous remercie pour cette question. Je ne suis pas sûr qu'il y ait un message dans cette Dame noire qui a été écrite alors que je finissais ma thèse. J'avais envie d'écrire autre chose, de voir si j'étais capable d'écrire un roman mais en même temps je ne songeais pas à écrire de la Littérature avec un L majuscule, donc la littérature de genre s'est assez rapidement imposée. Évidemment quelqu'un comme Iain Pears m'a influencé directement, j'en parle parce que je sais qu'il a été un illustre prédécesseur interviewé dans le cadre de précédentes journées de la FASP. Une influence qui s'est exercée à double titre : Iain Pears auteur de An Instance of the Fingerpost, qui traite aussi de la période sombre de la Révolution anglaise, mais aussi le Iain Pears moins sérieux, celui de la série des Jonathan Argyll qui se passe dans le monde de l'histoire de l'art. Sans doute, de façon souterraine ou explicite, trouverait-on des références à des œuvres littéraires, de Milton à Donne ou Burnes, mais elles sont pour ainsi dire des éléments consubstantiels d'une FASP universitaire. Donc effectivement de ce point de vue-là oui, c'est surtout une fiction à substrat professionnel aussi du fait qu'on écrit sur tout ce qu'on connaît, me semble-t-il. On m'a toujours appris quand j'étais étudiant, puis j'ai longtemps enseigné à distinguer l'auteur du narrateur, la vie de l'œuvre etc. toute la tarte à la crème proustienne et j'ai été fort surpris en passant de l'autre côté, en décidant d'écrire une histoire, d'écrire un roman, de constater, en tout cas en ce qui me concerne, qu'il fallait que je mette des choses que je connaissais, donc effectivement le monde universitaire s'est aussi imposé assez vite d'où le substrat professionnel universitaire. De ce point de vue-là c'est effectivement une fiction à substrat professionnel même si alors j'ignorais la notion. Le roman n'a pas été écrit pour se conformer à ce genre au moment où il était en train d'être théorisé dans les travaux de Michel Petit et dans le cadre du GREMUTS. Quant au Da Vinci Code, je ne sais pas. J'espère qu'il y a davantage de second degré dans La Dame Noire...

3 JLT : Vous avez aussi écrit pour être lu. Au cours de ces journées nous avons évoqué, à propos de la linguistique de l'énonciation, la visée du destinataire, nous aurions également pu parler en votre honneur de la grammaire de Port-Royal. Cela m'amène directement à ma deuxième question, pour qui avez-vous écrit ? Pour quel public ?

TG : Je n'avais pas de lecteur-type, implicite ou conscient dans ma démarche. J'ai surtout écrit pour savoir si j'étais capable d'écrire une histoire, d'écrire un roman. En même temps de fait, c'est vrai qu'étant donné que ce roman est peu diffusé, ce sont surtout des collègues, des universitaires, qui le lisent et qui, pour certains du moins, semblent l'apprécier. Dans quelle mesure est-il susceptible d'être lu et apprécié au-delà du milieu lié aux ultra-professionnels dont il est issu, c'est quelque chose dont je ne peux pas avoir d'idée très claire. 
$5 \quad$ JLT : Je pense quand même qu'ou bien vous vous êtes fait plaisir parfois ou bien vous aviez une petite idée derrière la tête. Pour qui a regardé ce que vous avez pu écrire par ailleurs je n'en veux comme exemple que les patronymes de vos personnages, noms et prénoms. Ce n'est certainement pas un hasard si votre héros contemporain se prénomme Arnaud, ce n'est pas non plus un hasard si le dernier patronyme à rallonge de Véronique rappelle un autre de vos écrits.

TG : Oui, alors, c'est classique, l'onomastique signifiante c'est quelque chose qu'on trouve dans la littérature. Arnaud ne rappelle pas tellement Port Royal, Arnaud Rudel c'est Arnaud Daniel et Geoffrey Rudel, je voulais qu'il ait un côté troubadour : l'amour de loin, les amours inaccessibles. De même Véronique, «image de vérité ", parce que c'est elle qui trouve l'essentiel de la vérité. C'est un livre que je me suis beaucoup amusé à écrire : quel plaisir de pouvoir rédiger sans mettre des notes de bas de page! Quel plaisir de pouvoir manipuler les faits, manipuler la vérité en toute impunité : dans quelle mesure le romancier historique a-t-il le droit, est-il fondé à transformer les faits? J'ai un ami qui s'est scandalisé parce que j'avais déplacé le tableau qui se trouve dans le couloir de la Sorbonne (je l'ai mis au fond de la bibliothèque), il m'a dit « tu n'as pas le droit de faire ça », je lui ai répondu «je fais exactement ce que je veux» et personne ne peut m'en faire grief au plan scientifique puisque ce n'est pas un ouvrage scientifique! C'est ce qu'on ne peut jamais se permettre quand on écrit dans le genre académique habituel.

7 JLT : Et, pour revenir à l'onomastique, que pouvez-vous nous dire sur Charity Backwater?

8 TG : Backwater est un personnage de Conan Doyle, c'est un aristocrate décadent. Et cela rappelle également backwards, on navigue en eaux troubles. Charity est un nom qui a été forgé sur les habitudes puritaines de donner des noms d'identités théologiques aux personnages. Charity c'est aussi la plus haute forme de l'amour et effectivement elle a un rapport très complexe à l'amour, à la fois l'amour de l'humanité, une volonté de faire avancer la société dans un sens qui nous paraitt terrifiant puisque c'est pour nous une espèce de théocratie puritaine effrayante, et du point de vue sexuel aussi, puisque c'est une antinomienne et qu'à l'époque on a beaucoup prêté aux puritaines de tendance antinomienne des comportements sexuels déviants, sans que les historiens puissent vraiment savoir si c'était vrai ou si c'étaient des critiques qu'on leur adressait.

9 JLT : De votre côté vous n'avez pas hésité à trancher pour les pratiques sexuelles. Quand on lit la nuit de noce, effectivement, on voit que vous avez pris une décision sur la vérité de ces pratiques!

10 TG : Oui, effectivement, mais on est dans le cadre d'une littérature de genre, et donc j'ai délibérément accepté toute une série de stéréotypes, de passages obligés et donc j'ai obéi à tout ce que j'identifiais comme relevant de la poétique de la FASP, ou plutôt du thriller, du polar, et donc il y a effectivement la présence du sexe. J'étais condamné à introduire quelques pages un peu chaudes. Mais, malgré tout, un tel épisode s'inscrit dans une perspective historique. On pourrait me rétorquer que certains historiens remettent en cause la réalité de ces pratiques déviantes attribuées aux nonconformistes mais elles restent malgré tout une possibilité ouverte par l'histoire.

11 JLT : Oui, il ne s'agit que de quelques pages mais elles aident bien à comprendre les rapports compliqués qui se sont établis entre les deux époux.

Pour revenir à un sujet un peu plus traditionnel, le roman académique est un genre qui 
se porte bien dans le monde anglo-saxon, nous avons déjà eu l'occasion d'en parler, avec des auteurs comme Snow, Amis, Lodge, ou moins sérieusement Tom Sharpe. En France le roman académique est quelque chose de relativement rare. Qu'est-ce qui, selon vous, peut expliquer une telle différence entre nos deux cultures?

TG : C'est vrai que je me suis rendu compte que j'écrivais un roman académique sans l'avoir vraiment prémédité et je me suis demandé s'il y en avait d'autres en France. Comme par hasard le cadre de mon roman n'est pas la France! C'est un roman académique qui se passe à Cambridge. Pourrait-on écrire le même type de roman mais situés sur le campus de Mont St Aignan ou celui de Grenoble? il faudrait essayer pour savoir si c'est possible mais la vie universitaire ici est tellement différente... en Angleterre les campus sont des mondes clos où on travaille, où on vit, où on dort, où on existe, où on se distrait, où on fait plein de choses...

JLT : Où on se massacre!

14 TG : Où on se massacre au sens propre, mais seulement dans mon roman! Au sens figuré, en revanche, je ne sais pas... Je pense malgré tout que le cadre du campus anglosaxon se prête davantage aux intrigues policières classiques, qui exigent des espaces fermés. Il y a eu, par exemple, des romans policiers qui se déroulent dans l'enceinte de l'ENS, mais précisément cette école est un lieu clos.

15 JLT : Un de mes collègues, Professeur de Lettres Classiques comme vous, est en train d'écrire un roman policier dont l'action se déroule à la Sorbonne, la victime, un professeur de latin se fait tuer à coups de Gaffiot! Nous aurons peut-être l'occasion d'en parler lorsqu'il sera publié. Mais revenons à la FASP. Nous avons déjà dit que la FASP, phénomène anglo-saxon surtout, était traduite en plusieurs langues. Lorsque vous avez écrit, compte tenu de différents jeux de mots, des allusions, avez-vous pensé au fait que vous pouviez éventuellement être traduit en anglais et, si oui, qu'est-ce qui, selon vous, posera le plus de difficultés au traducteur?

16 TG : C'est une question que je ne me suis absolument jamais posée. C'est un livre assez particulier dans la mesure où c'est un livre écrit par un Français, en français, mais qui se déroule entièrement en Angleterre. Le livre comporte donc des réalités anglosaxonnes qui ne poseraient pas de problèmes à traduire, mais qui m'en ont posé à moi parce que je me suis trouvé dans la position d'un traducteur! Par exemple le titre original de ce roman est un titre anglais, Ghost Tour. Vous connaissez ces promenades, comme on en fait à Edimbourg ou ailleurs, où on emmène des touristes visiter les endroits réputés hantés de la ville. Non seulement il n'y a pas d'équivalent français, mais comment traduire ça d'une façon élégante en français? Promenade aux fantômes ? Ça ne marchait pas. Rien de ce que j'ai proposé à mon éditeur ne lui plaisait. De même Formal Hall ! On sait tous ce qu'est un Formal Hall à Cambridge ou à Oxford mais il n'y a pas d'équivalent en France alors que dire ? Repas en toge ? Repas officiel ? Et c'est cela qui m'a posé le plus de problèmes. Il y a aussi de petites facéties liées à la langue : ainsi Véronique, quand elle est émue, emploie des anglicismes. J'ai souvent remarqué cette déformation professionnelle chez les anglicistes et elle m'amuse beaucoup!

Je n'ai pas du tout répondu à votre question !!!

17 JLT : Pas du tout en effet! Mais partons du principe que vous allez être traduit en anglais. Selon vous, votre public britannique, restons au Royaume-uni au moins dans un premier temps, sera-t-il capable d'apprécier votre ouvrage? 
18 TG : Il n'est pas totalement impossible que la traduction en anglais se fasse, mais le projet est encore lointain. J'ai été surpris de constater qu'un universitaire de Cambridge a pu apprécier ce roman, et ce malgré l'image que je donne de Cambridge. Cambridge est une ville qui me fascine ; je voulais faire un roman d'atmosphère et je m'aperçois que j'en donne une image absolument désastreuse et catastrophique qui n'était absolument pas mon idée initiale.

19 JLT : Mais comme vous êtes très prudent, vous avez situé votre roman à All Souls qui est le nom d'un College d'Oxford et non de Cambridge !

20 TG : Oui, c'était aussi un choix fonctionnel, All Souls était bien en rapport avec une histoire de fantômes, cela fait penser à Halloween...

21 JLT : À ce propos je vous remercie, vous êtes le premier à me faire remarquer que les chapeaux des Puritains étaient des troncs de cônes des chapeaux de sorcière!

TG : À partir de 1640, dans le contexte tourmenté de la Guerre Civile il y a une recrudescence de la chasse aux sorcières et effectivement il y a une assimilation des membres de sectes radicales aux sorcières. Et ce chapeau est le même que celui des Cavaliers, sauf que l'un a des plumes et l'autre n'en a pas.

JLT : Est-ce que votre participation à nos journées d'études peut influencer, sinon votre inspiration, du moins le traitement de votre prochain ouvrage puisqu'il y a une suite en gestation.

24 TG : La préparation de l'HDR n'est pas vraiment compatible avec l'écriture d'un roman policier, mais si j'en écris un autre je pense qu'il se passera en France, toujours dans le contexte universitaire, peut-être avec les mêmes personnages. Et ce sera peut-être aussi davantage de la FASP, c'est ce que j'ai pu retirer des échos que m'ont donnés certains lecteurs. Ce qui m'a aussi surpris quand j'ai écrit, et j'ai trouvé ça dans les différents écrits sur la FASP, c'est que la satire est une composante du roman académique: spontanément le ton était bien davantage satirique contre l'université que je ne le voulais, comme s'il y avait une sorte de pression du genre académique, pression à laquelle j'ai dû résister autant que possible. Je ne voulais pas faire du Tom Sharpe!

25 JLT : Pour rester dans le sujet académique, ne craignez-vous pas d'être handicapé dans votre carrière par le fait que vous allez devenir, du moins je vous le souhaite, un auteur à succès dans un genre considéré comme mineur, que ce soit à tort ou à raison d'ailleurs. Ne craignez-vous pas soit le mépris de vos pairs, soit le monstre aux yeux verts de la jalousie?

26 TG : À la surprise d'un de mes amis j'ai effectivement publié sous mon vrai nom. Tant que je serai publié à 150 exemplaires je n'exciterai pas l'envie et la jalousie dévorantes de mes collègues. Je n'en suis pas encore au jour où je serai vraiment un auteur de bestsellers, un paperback writer comme chantaient les Beatles.

JLT : Outre le passage obligé par le sexe, quelles sont les autres recettes que vous avez utilisées pour l'écriture?

TG : On dit qu'un vrai magicien ne dévoile jamais ses secrets! En fait, j'ai fait comme pour l'écriture scientifique, j'ai essayé de travailler méthodiquement. J'ai donc étudié un genre particulier qui n'existe pas en France, je veux parler des manuels de creative writing, et j'ai beaucoup appris à leur lecture. J'ai donc appris toutes sortes de recettes que j'ai essayé d'appliquer très consciencieusement en bon élève. Puis il y a aussi des 
procédés tirés de la lecture de romans, de thrillers, de polars. C'était aussi un exercice de style, j'ai essayé de jouer le jeu des conventions, comme il convient pour une livre relevant de la littérature populaire.

29 JLT : Je voudrais savoir si vous avez poussé la conscience professionnelle jusqu'à composer un véritable message codé.

TG : Bien sûr, j'ai utilisé la méthode de cryptologie de Blaise de Vigenère, et l'on trouve sur Internet des petits programmes qui codent et décodent ! Il fallait un message codé, après Leblanc et Arsène Lupin...

31 JLT : Poe et son scarabée...

32 TG : Oui, il y a une allusion à La lettre volée. Tout cela faisait partie du jeu.

33 JLT : J'en reviens donc à ma question initiale, vous aviez quand même un certain lectorat en tête : pour que l'on puisse apprécier pleinement votre ouvrage au-delà de l'intrigue il est nécessaire de posséder une bonne culture littéraire

34 TG : J'avais eu des contacts avec des éditeurs de polars, et en particulier Belfond, l'éditeur de Pears. L'on m'a finalement répondu que le roman est un peu trop ambitieux pour leur lectorat. J'ai utilisé toutes les techniques du roman populaire et, au final, ce n'est pas un roman populaire, ce qui là aussi m'a surpris. J'ai voulu faire quelque chose de très sérieux sur le plan historique et en même temps reproduire des procédures universitaires. L'enquête se déroule selon la méthode qui est celle de la recherche universitaire. Véronique recommande à Arnaud de consulter le DNB, mais comme il ne connaît pas ce dictionnaire biographique, Véronique doit le lui expliquer. On retrouve ainsi le mode de dialogue en trois temps que Shaeda Isani nous a présentés et analysés, à savoir la mention d'une réalité professionnelle par un protagoniste, que son interlocuteur ne connaît pas, et qu'il faut lui expliquer. Lors du dénouement, les conclusions de l'enquête sont apportées pendant un colloque. De ce point de vue-là, $L a$ Dame noire relève de la FASP : l'ancrage universitaire concerne non seulement le décor, mais aussi les méthodes de la recherche.

35 JLT : Une toute dernière question puisque nous arrivons à la fin du temps qui nous est imparti. Est-ce que le petit montage que l'on trouve à propos de votre ouvrage sur YouTube a été voulu par vous ou par votre éditeur?

36 TG : Mon éditeur est un tout petit éditeur associatif qui prend des risques et m'a demandé de faire de la promotion, de jouer le jeu, et comme j'ai quelques compétences en informatique je me suis une fois de plus amusé. J'ai trouvé que c'était bien dans le ton de ce que je voulais faire c'est-à-dire sous la forme de bande-annonce de film populaire... ce qui a consterné bon nombre de mes amis !

37 JLT : Tony Gheeraert, je vous remercie d'avoir bien voulu répondre à ces questions. 


\section{NOTES}

1. La Dame Noire, introuvable sous sa forme originelle, est maintenant disponible aux Éditions Le Pommier sous le titre Le Code de Cambridge (Paris, 2010, coll. « Romans et Plus », postface de Pierre-Alain Fouque et Charles Bouillaguet, 504 p., format 135 x 200 cm, ISBN 978-2-7465-0465-3). 